Boise State University

ScholarWorks

Mathematics Faculty Publications and

Presentations

$5-2017$

\title{
An Exploration of a Quantitative Reasoning Instructional Approach to Linear Equations in Two Variables with Community College Students
}

\author{
Paul T. Belue \\ College of Western Idaho \\ Laurie Overman Cavey \\ Boise State University \\ Margaret T. Kinzel \\ Boise State University
}

This is the peer reviewed version of the following article:

Belue, Paul; Cavey, Laurie Overman; and Kinzel, Margaret T. (2017). An Exploration of a Quantitative Reasoning Instructional Approach to Linear Equations in Two Variables With Community College Students. School Science and Mathematics, 117(5), 183-193.

which has been published in final form at doi: 10.1111/ssm.12223. This article may be used for non-commercial purposes in accordance with Wiley Terms and Conditions for Self-Archiving. 
This is an author-produced, peer-reviewed version of this article. The final, definitive version of this document can be found online at School

Science and Mathematics, published by Wiley. Copyright restrictions may apply. doi: 10.1111/ssm.12223

\title{
An Exploration of a Quantitative Reasoning Instructional Approach to Linear Equations in Two Variables with Community College Students
}

\author{
Paul T. Belue \\ Assistant Professor \\ Mathematics \\ College of Western Idaho \\ Dr. Laurie Overman Cavey \\ Associate Professor \\ Mathematics \\ Boise State University \\ Dr. Margaret Kinzel \\ Associate Professor \\ Mathematics \\ Boise State University
}

\begin{abstract}
In this exploratory study, we examined the effects of a quantitative reasoning instructional approach to linear equations in two variables on community college students' conceptual understanding, procedural fluency, and reasoning ability. This was done in comparison to the use of a traditional procedural approach for instruction on the same topic. Data were gathered from a common unit assessment that included procedural and conceptual questions. Results demonstrate that small changes in instruction focused on quantitative reasoning can lead to significant differences in students' ability to demonstrate conceptual understanding compared to a procedural approach. The results also indicate that a quantitative reasoning approach does not appear to diminish students' procedural skills, but that additional work is needed to understand how to best support students' understanding of linear relationships.
\end{abstract}

\section{Introduction}

Recommendations made by the American Mathematical Association of Two Year Colleges (AMATYC), call for community college instructors to teach mathematics "in the context of realistic, understandable, applied problems that help students develop an appreciation of the nature, history, and usefulness of the discipline” (Blair, 2006, p. 10). The underlying goal is for students to understand mathematics, develop problem solving skills, and develop conceptual understandings (Blair, 2006, p.6 \& p. 54).

Similar principles and standards for teaching mathematics are called for more broadly in the mathematics education community (Leinwand, 2014). Citing classroom research from the last 30 years, Leinwand et al. (2014) assert that mathematics teachers should engage "students in solving and discussing tasks that promote mathematical reasoning and problem solving” (pp. 10- 11). Moreover, Leinwand et al. identified two strands that constitute mathematical proficiency: conceptual understanding and procedural fluency, where procedural fluency is to be built upon "a foundation of conceptual understanding so that students, over time, become skillful in using procedures flexibly as they solve contextual and mathematical problems” (Leinwand et al., 2014, pp. 9-10).

But how can community college mathematics instruction be structured to effectively teach quantitative problem solving? Research suggests using an instructional approach with a focus on students learning how to identify quantities in real world situations and how to reason with these quantities to produce new quantities (Thompson \& Thompson, 1992; Carlson et al., 2013; Ellis, 2007a; Simon \& Blume, 1994; Lobato \& Thanheiser, 2002) Others have made the 
This is an author-produced, peer-reviewed version of this article. The final, definitive version of this document can be found online at School

Science and Mathematics, published by Wiley. Copyright restrictions may apply. doi: 10.1111/ssm.12223

case for how understanding, identifying, and reasoning with quantities is foundational to all mathematics, especially in developing the mathematical ways of thinking needed to solve mathematical problems encountered in real life, as well as within mathematics and other disciplines (Dougherty et al., 2010).

Further, there is broad consensus that conceptual knowledge supports procedural knowledge but there is still debate about what order procedural knowledge and conceptual knowledge should be taught (Rittle-Johnson et. al, 2015). For example, Schwartz and Martin (2004) showed that conceptually-based activities are effective for preparing students to learn from lectures and other learning resources. However, other research shows children first learn procedures for solving problems and then after repeated practice solving problems extract conceptual knowledge (Fuson, 1988; Karmiloff-Smith, 1992; Siegler \& Stern, 1998). Still others have shown that the teaching and learning of conceptual understanding and procedural skill build on each other in an iterative or cyclical process (Rittle-Johnson, 2001).

\section{Purpose of This Study}

This study examined an experimental model of instruction for teaching linear equations in two variables that focused on using real world contexts, developing students' mathematical reasoning skills with quantities, and building procedural fluency upon a foundation of conceptual understanding (hereafter called a quantitative reasoning approach). An intervention group was taught a unit on linear equations in two variables using a quantitative reasoning approach, and a comparison group was taught a unit on the same topic using a traditional procedural approach, which emphasized learning and practicing step by step solution methods to solving routine problems. Data were collected on a common unit test for the two groups to determine:

1. What does the comparison of unit assessment data between the two instructional groups indicate about the relationship between conceptual knowledge and procedural knowledge in the teaching of mathematics to community college students?

2. How does the mathematical performance and reasoning ability for students in the two instructional groups compare?

\section{Background}

\section{Quantitative Reasoning}

Following Thompson and Thompson (1992), we use quantity when referring to an individual's conception of a measurable quality of an object or event. A quantity consists of an object or event, a quality of the object or event, an appropriate unit or dimension, and a process for assigning a numerical value to the quality (Thompson, 1994). Measurement of a quality (or attribute) involves making a multiplicative comparison between the quality of interest (such as the width of a rectangular floor) and the unit (such as a meter). However, making a direct measurement is not necessary to conceive of a quantity. This is because "quantities are constituted in people's conceptions of situations, rather than the objects or situations themselves" (Ellis, 2007a, p. 440).

Simply put, quantitative reasoning is reasoning about things and their attributes (Thompson \& Thompson, 1992). Two types of quantitative reasoning relevant for beginning algebra students learning about linear equations in two variables are: covariation reasoning and correspondence reasoning (Jacobson, 2014). Covariation reasoning involves analyzing how two quantities vary in tandem, while correspondence reasoning involves identifying a functional relationship between two quantities. Both forms of reasoning are crucial to developing an understanding of function. Covariation reasoning is used to examine how one quantity changes in relation to changes in another quantity. Correspondence reasoning is used to determine how one quantity can be determined by the other. In the case of linear functions, noticing that two quantities co-vary with a constant rate of change is an important aspect of understanding the slope-intercept form of a linear equation (Jacobson, 2014; Carlson et al., 2002).

Students deficient in quantitative reasoning are poor at algebraic problem solving (Thompson, 1988). When students name quantities by their numerical value only instead of by the name of the measurable quality of the object (e.g. " 100 dollars" instead of "Paul's savings") they do not distinguish between the quantity and its measure. This leads to difficulty identifying quantities that have an unknown value and deciding which operation to perform when determining a third quantity. 
This is an author-produced, peer-reviewed version of this article. The final, definitive version of this document can be found online at School Science and Mathematics, published by Wiley. Copyright restrictions may apply. doi: 10.1111/ssm.12223

Comparing quantities multiplicatively to form a ratio is another special type of quantitative reasoning called proportional reasoning. Ratios are formed to measure how many times larger or how many times smaller a quantity is than another (multiplicative comparison), or the intensity of a quantity compared to another (composed unit) (Lamon, 2012). A ratio-as- measure task requires students to identify a ratio as an appropriate measure of a given attribute and to create that ratio (Simon \& Blume, 1994). When a ratio is not given at the outset but rather is developed by the classroom as a useful mathematization of the situation, greater understanding of the quantity and of ratio are likely to result. When students are given a ratio as a measure of a given attribute they do not necessarily see a need for a ratio to measure the attribute and why the ratio is appropriate. As Harel's (2001) Necessity Principle asserts, students are most likely to learn if they see an intellectual need for what we intend to teach them.

Using ratios as measures can be a difficult transition for students (Simon \& Blume, 1994; Lobato \& Thanheiser, 2002; Smith et al., 2013). Students may struggle to isolate attributes in a situation that a ratio measures from other attributes (Lobato \& Thanheiser, 2002; Lobato et al., 2010, p. 23). Students may also struggle with using ratios to compare attributes. For example, research shows that students struggled to know whether changing the length of the platform at the end of a ramp affected the slope of the ramp or not (Lobato et al., 2010). On the other hand, Ellis (2007a) found that when a group of middle school students focused on quantities in real world contexts instead of numbers in tables, they searched for a new relationship between the quantities, experienced the necessity to create a ratio as a measure, and understood the ratio remains constant even when the quantities changed. Ellis (2007a) named reasoning leading to the construction of a ratio as emergent-ratio quantitative reasoning.

\section{A Quantitative Reasoning Approach}

A quantitative reasoning approach to instruction includes exploring with students how changing quantities affect an emergent quantity, giving students time to reflect on a problem's context, and helping students identify a quantity by the name of the measurable attribute. Simply placing students in a quantitatively rich situation does not necessarily guarantee students will develop correct understandings of the concepts (Ellis, 2007a; Moore \& Carlson, 2012). Instead, the type of quantitative reasoning students engage in is what results in productive mathematical understandings. Ellis (2007a) suggested students engage in activities such as: (a) Exploring how changing one or both initial quantities will affect the emergent quantity, (b) Determining how to adjust the initial quantities while keeping the emergent quantity constant, and (c) Determining how to double, halve, or otherwise manipulate the emergent quantity in relationship to the initial quantity. Students also need to be clear and specific on what is being measured to eventually write meaningful expressions and formulas (Carlson, Oehrtman, \& Moore, 2013).

Ellis (2007a) studied the use of a quantitative reasoning instructional approach by examining the generalizations of middle school students taught linear functions in two different learning and teaching environments. In the first environment, students were taught linear functions using an approach that emphasized number pattern finding from tables of linear data, procedures, skills, and direct computational questions and responses. In the second environment, students were taught linear functions using an approach that emphasized quantitative reasoning: i.e. exploring quantities and their relationships in two real world situations about linear growth. Ellis (2007b) observed the work of seven students from each learning environment in the classroom and through interviews.

Students' generalizing actions and reflection generalizations were counted and placed into the categories of Ellis' generalization taxonomy (Ellis, 2007b). Two types of generalizations made by students are particularly relevant for our work: statements of continuing phenomena and a statement of global rules (Ellis, 2007b). Statements of continuing phenomena were statements made by the students identifying a dynamic property extending beyond a specific instance such as "every time x goes up 1, y goes up 5." A statement of global rules is a generalization of a relationship such as "If the rate of change stays the same, the data are linear" (Ellis, 2007b).

Although the number of global rules statements made by the number-pattern students was more than the number made by the quantitative reasoning students, analysis of the interview data showed that the correctness of the global rules statements favored the quantitative reasoning students (Ellis, 2009). The number-pattern students incorrectly generalized that a table of data representing a situation is linear only if the numbers down a column in the table increased or decreased by the same amount between rows. They appeared to make this overgeneralization because all the tables they examined had this uniform quality. When they encountered a non- uniform table that represented a linear situation, they struggled and declared it nonlinear. The quantitative reasoning students extended their 
This is an author-produced, peer-reviewed version of this article. The final, definitive version of this document can be found online at School Science and Mathematics, published by Wiley. Copyright restrictions may apply. doi: 10.1111/ssm.12223

understanding of linearity to both uniform and non-uniform tables that represented real world situations. Interestingly, the quantitative reasoning students made a greater number of statements of continuing phenomena, attending to the covarying nature of the quantities (Ellis, 2007b).

Using a quantitative reasoning approach shows promise for developing students' algebraic reasoning skills. This exploratory study is an initial attempt to investigate the effects of such an approach in a community college setting.

\section{Methodology}

\section{Participants \& Design}

The participants of this study ( $\mathrm{n}=71$ ) were beginning algebra students at a community college in the northwest. Thirtyfour of the participants were taught a unit on linear equations in two variables using a traditional procedural approach (comparison group) and 40 participants were taught a unit on the same topic using a quantitative reasoning approach (intervention group). The students were placed in the beginning algebra course based on their placement test score or by earning a $C$ or better in a pre-requisite Prealgebra course. No further selection criteria were applied other than asking for voluntary participation in accordance with human subjects protocol. For the comparison group, 34 of the 48 enrolled students agreed to participate; 37 of the 50 enrolled students in the intervention group participated. The lead author was the instructor for both groups (the comparison group one semester and the intervention group the following semester). At the end of instruction (four days for each group) both groups took the same unit assessment with ten items designed to assess procedural understanding (procedural items) and ten items designed to assess conceptual understanding (conceptual items). The responses of the two groups on the unit assessment items were then analyzed to compare performance and reasoning ability between the comparison and intervention groups.

\section{Instructional Approaches}

Lessons were developed by the lead author who was experienced in using the traditional procedural approach but was using the quantitative reasoning approach for the first time. The lead author worked closely with the second author to develop lessons for the intervention group, and the third author provided feedback on lesson enactment early during the unit.

The traditional procedural approach consisted of the teacher explaining a step-by-step solution method to solving a routine non-contextual problem, demonstrating the solution method, and then having the students practice. For example, on the first day the instructor explained how to determine if values of the variables are solutions to a linear equation in two variables, then demonstrated how to replace the variables in the linear equation with their respective values, simplify the equation, and see if a true statement resulted. He then gave the students a different linear equation in two variables with different values for the variables and had the students practice the step-by-step procedure. See Belue (2015) for detailed lesson plans and learning objectives. The students were then assigned similar homework problems to practice outside of class using online course software. Problems for instruction and homework were based on a beginning algebra developmental math textbook (see Trigsted et al., 2014).

The quantitative reasoning approach consisted of the teacher explaining and helping students understand a quantity and linear equation in two variable concepts in real world contexts and then engaging students in tasks on a worksheet to further develop reasoning ability. For example, on the first day the lesson began with the teacher explaining the four components of a quantity, giving an example of a quantity in the classroom (the length of the floor in feet), and asking students to identify other quantities associated with the floor. The teacher then led a discussion on attributes of the floor that are not quantities followed by the teacher walking across the floor of the classroom and having the class identify the quantities involved in the walking event. The teacher then introduced the terms variable quantity and fixed quantity. Finally, the teacher then walked across the floor again and had the students measure his walking rate. The students then wrote down the walking rate and worked through proportional reasoning tasks with the rate on their worksheets in small groups. These types of activities, involving physical demonstrations of concepts and using different types of reasoning (quantitative, proportional, correspondence, and covariation) continued through day three of instruction. The problems and exercises on the worksheet were based on curricula designed to engage students in different types of reasoning (see Carlson et al., 2013, \& Lappan et al., 2009). On day four the teacher conducted a review for the unit assessment which included addressing standard procedures for solving non-contextual problems like the ten procedural problems on the unit assessment. 
This is an author-produced, peer-reviewed version of this article. The final, definitive version of this document can be found online at School Science and Mathematics, published by Wiley. Copyright restrictions may apply. doi: 10.1111/ssm.12223

\section{$\underline{\text { Unit Assessment }}$}

A twenty-item unit assessment, with ten procedural items and ten conceptual items, was constructed to compare performance between the two groups. The ten procedural items were short answer questions designed to align with previous assessments in the course and had no real world context. They were routine problems similar to the problems the comparison group encountered during class and were intended to assess the students' ability to perform a step-bystep solution process (memorizable) to answer the question. The ten conceptual items on the other hand could not be answered by performing a step-by-step solution procedure and were intended to assess conceptual understanding and reasoning abilities associated with linear equations with two variables. They were ten short answer questions about a real world situation of a person filling a swimming pool with water at a constant rate. They required the student to first correctly identify constant and varying quantities, then construct tables, graphs, and equations that model the situation. Items 15 and 17 asked students to explain how these representations tell you the situation is or is not linear. Thus, these items required students to provide evidence of conceptual understanding and reasoning ability to completely answer the questions. Detailed rubrics for assessing students' work for the procedural and conceptual items were applied to both groups. (See Belue (2015) for the complete list of assessment items, rubrics, and the development processes.) For example, the rubric for item 15 (asking how the table they constructed tells you the situation is linear) assigned a score of $0,1,2,3$, or 4 for different levels of specificity with respect to student reasoning exhibited by their written response. To earn a 4, students needed to give a correct explanation (using either covariation or correspondence reasoning) and make explicit reference to data in the table.

The assessment items and rubrics were initially developed by the first author, with the second and third authors performing expert reviews and offering feedback. It is beyond the scope of this exploratory study to pursue validity issues beyond the expert analysis of whether items were constructed to capture the intended reasoning by students (both procedural and conceptual). Rubrics were developed in a similar fashion, relying heavily on the literature on quantitative reasoning to identify anticipated reasoning categories associated with the conceptual items.

\section{$\underline{\text { Data Collection }}$}

Students in both groups had 75 minutes to complete the unit assessment. The twenty questions were graded by assigning the point values for each question as prescribed in the assessment rubrics (Belue, 2015) These raw scores were then used to perform the quantitative analysis. In addition, we used qualitative methods to analyze students' responses to questions 15 \& 17. These two items provided an opportunity to assess students' conceptual understanding of connections between representations of linear functions and defining aspects of a linear relationship between two quantities.

\section{Quantitative Analysis}

Raw scores based on the rubric for each question on the unit assessment were converted to scaled scores. For example, if a student received a raw score of three points out of four for an item according to the rubric the score was changed to 7.5 out of 10 , or $75 \%$ (since raw scores for each item varied from two to four points). Scaled score means were computed for each item and each instruction group to conduct an item by item analysis on the set of scaled score means. The set of scaled score means was then statistically tested using a two-tailed t-test to determine if the two population means (one for the comparison group and one for the intervention group) were the same. They were then statistically tested using a one tailed t-test (if not the same) to determine if the mean of one population was greater than the other.

\section{Qualitative Analysis}

The qualitative analysis started with the lead author reading each student's response to items 15 and 17 on the unit assessment and scoring the responses into categories of similar explanations. This resulted in a total of ten initial categories for item 15 and a total of eleven initial categories for item 17. The students' responses and initial categories of similar explanations were then examined again by all three authors to determine how they related to covariation and correspondence reasoning. Based on this analysis and the combining of initial categories that originally separated incorrect from correct responses, four final categories of student thinking about linearity emerged. Of interest was students’ ability to make correct generalizations (i.e. global rules statements) about linearity and statements of 
This is an author-produced, peer-reviewed version of this article. The final, definitive version of this document can be found online at School Science and Mathematics, published by Wiley. Copyright restrictions may apply. doi: 10.1111/ssm.12223

continuing phenomena involving covariation reasoning (Ellis, 2007b). We based the coding on the types of reasoning involved in determining a linear relationship even though students' responses were not always completely correct. The following is a description of the four categories.

Covariation Reasoning. Student responses in this category explained that the table or graph showed the pool filled at a constant rate using explicit references to the quantities involved in the given situation. For example, one student response placed in this category was "The table tells you it is a linear relationship by being consistent. For every 3 seconds, 1 gallon is added" and another student response was, "It is linear b/c for every gallon added the seconds go up by 3 , the rate is always the same.” These types of responses were considered correct global statements about linearity and statements of continuing phenomena involving covariation because they explicitly addressed how the two quantities changed together at a constant rate (Ellis, 2007b).

Correspondence Reasoning. Student responses in this category explained that the table or graph showed there was an explicit rule that related the two quantities (volume and time). For example, a student response placed in this category was, "It is a constant growth that does not vary. The table shows this because no matter what you plug in for s, g will always be $1 / 3$ of that." These types of responses used correspondence reasoning because they explicitly addressed how the quantities were related by a functional rule.

Beginning Quantitative Reasoning. Student responses in this category were somewhat superficial explanations of how the quantities in the situation were related (using indications of either covariation or correspondence reasoning). Students attended to the quantities but without specific reference to how the quantities were related. For example, a student response in this category was, "The table shows it is linear because as the seconds increase, so too does the value of gallons" and another student response was, "It is a linear relationship because as the time increases the number of gallons in the pool also increases." These types of responses did not explicitly attend to how the quantities were linearly related.

Non-Quantitative Reasoning. Student responses in this category explained that the situation was linear because the table or graph was constant but with no explanation of what feature associated with the table or graph was constant. For example, a student response in this category was, "It is a linear relationship because there are consistent measurements" and another student response was, "it is a linear equation because is constant." This category also included responses that explained the situation was linear because the graph was a straight line. For example, one student wrote "It shows it is a linear relationship because the line is a straight line." These types of responses demonstrated no quantitative reasoning because they did not reference specific quantities in their explanations and did not show any evidence of covariation or correspondence reasoning.

The responses in these four categories were then analyzed and compared between the comparison group and intervention group to identify similarities, differences, and themes in the students' thinking about linearity. A test for proportions was conducted to determine if performance was significantly different in each of the four categories.

\section{Results}

A quantitative and qualitative analysis between the intervention and comparison group at the individual item level of the procedural and conceptual items on the unit assessment was conducted to compare the students' performance and reasoning ability.

\section{Quantitative Analysis}

Procedural Items. The scaled score means for each of the 10 procedural items for the comparison group and the intervention group are listed in Table 1. A brief description of each item is also included to illustrate the range of problem types. Notice that the comparison group $(n=34)$ had a $10 \%$ or greater scaled score mean than the intervention group ( $\mathrm{n}=37$ ) on three of the 10 procedural items (items 4, 6, and 10). The comparison group and intervention group had about the same scaled scores (performance difference less than 10\%) on the remaining seven procedural items. Both the comparison and intervention group had low scores ( 0.62 and 0.56 respectively) on item 9 . A two tailed t-test conducted on the scaled score means of the 10 procedural items showed there was no difference in performance between the comparison and intervention group $(p>0.05)$. 
This is an author-produced, peer-reviewed version of this article. The final, definitive version of this document can be found online at School Science and Mathematics, published by Wiley. Copyright restrictions may apply. doi: 10.1111/ssm.12223

$<$ Insert Table 1 and Figure 1 here.>

Conceptual Items. The scaled score means for each of the 10 conceptual items for the comparison and intervention groups are listed in Table 2. A brief description of the conceptual items is provided to illustrate the range of problem types. All problems were tied to a single contextual situation involving filling a pool with water. The intervention group $(\mathrm{n}=34)$ had a $10 \%$ or greater scaled score mean than the comparison group $(\mathrm{n}=37)$ on 9 out of 10 of the conceptual items (11-13, and 15-20). A two-tailed t-test conducted on the scaled score means of the 10 conceptual items alone showed there was a difference in performance between the comparison and intervention group $(p<0.05)$. Further, a one tailed t-test on the scaled score means of the 10 conceptual items showed the intervention group performance was greater than the comparison group $(p<0.010)$.

< Insert Table 2 and Figure 2 here.>

The greatest differences in scaled score means on the conceptual items were a $43 \%$ greater mean on item 20, 31\% greater mean on item 11 , and a $23 \%$ greater mean on item 17 . The distribution of the scaled scores for each of these items and for each instructional group is displayed in Table 3 via quartile scores. On item 11, at least $50 \%$ of the intervention group had a scaled score mean that was 1.0 compared to less than $33 \%$ of the comparison. On item 20, at least $50 \%$ of the intervention group had a scaled score mean that was 0.75 or greater compared to less than $33 \%$ of the comparison. For each item, the median scaled score mean was greater for the intervention group compared to the comparison group. The greatest difference was the difference in medians for item 11, which had a difference of 0.67.

$<$ Insert Table 3 here.>

Item 14 was the only item where the comparison group and intervention group scored the same. Items 20 and 17 were the first and second most difficult conceptual items for students respectively (item 20 had a combined score of 0.43 and item 17 had a combined score of 0.48 ). Item 12 also had a low combined scaled score of 0.49 .

Additional results. A two-tailed t-test conducted on the scaled score means of the 10 procedural items and 10 conceptual items combined showed there was no difference in performance between the comparison and intervention group $(p>0.05)$. For the procedural items, three out of the top four most difficult items involved the concept of slope and two out of the four most difficult items involved graphing. For the conceptual items, three out of five of the most difficult conceptual items involved explaining concepts from a table, graph, and equation. Of note, the graphing item (16) was also difficult, having a scaled score mean similar to the scaled score mean for the graphing items in the procedural items (0.66 vs. 0.72$)$.

Qualitative Analysis

Categories and Student Response Counts. As explained in the methodology section, the categorization and merging of initial categories of explanation by students on question 15 and 17 according to their types of reasoning led to four categories of student thinking about linearity.

Table 4 shows the counts for student responses in the four categories of student thinking about linearity on items 15 and 17 of the unit assessment. There were a total of 31 responses from the comparison group and 35 responses from the intervention group (out of 71 participants) on item 15, and a total of 31 responses from the comparison group and 36 responses from the intervention group (out of 71 participants) on item 17 (one student in the intervention group did not respond to item 15 but did respond to item 17).

$<$ Insert Table 4 here.>

Student Response Counts Summary and Analysis. Seven of the 31 responses (23\%) in the comparison group and fourteen of the 35 responses (40\%) in the intervention group on item 15 were placed in the covariation reasoning category (e.g. "It is linear b/c for every gallon added the seconds go up by 3 , the rate is always the same"). A comparison of proportions test showed this difference was not statistically significant $(p=0.1324)$. Five of the 31 responses $(16 \%)$ in the comparison group and eleven of the 36 responses (31\%) in the intervention group on item 17 were placed in the covariation reasoning category. A comparison of proportions test showed this was not statistically significant $(p=$ $0.1704)$. 
This is an author-produced, peer-reviewed version of this article. The final, definitive version of this document can be found online at School Science and Mathematics, published by Wiley. Copyright restrictions may apply. doi: 10.1111/ssm.12223

Two of the 31 responses in the comparison group and one of the 35 responses in the intervention group on item 15 were placed in the correspondence reasoning category (e.g. "The table shows this because no matter what you plug in for s, $\mathrm{g}$ will always be $1 / 3$ of that"). A comparison of proportions test showed this was not statistically significant ( $p=$ 0.4874). Two of the 31 responses in the comparison group and none of the 36 responses in the intervention group on item 17 were placed in the correspondence reasoning category. A comparison of proportions test showed this was not statistically significant $(p=0.1246)$.

Although the student responses to items 15 and 17 that were placed in the correspondence reasoning category did not have the correct equation for the situation, there were five comparison students and four intervention students who wrote the correct equation for the situation on item 18 of the unit assessment. This item asked students to write an equation that modeled the situation. However, overall performance on item 18 was poor with the comparison group having a scaled score mean of 0.48 and the intervention group having a scaled score mean of 0.64 .

The poor performance by students on item 18 may have been related to performance on item 12 . This item asked students to identify variable quantities in the situation. The quantitative analysis on this item showed that students had difficulty identifying the variable quantities (scaled score mean was 0.43 for the comparison group and 0.54 for the intervention group). An interesting theme observed in the qualitative analysis is a number of students identified a rate as a varying quantity. Ten of the 31 responses in the comparison group and twenty-two of the 36 in the intervention group claimed that a rate is a varying quantity. This apparent confusion about the varying quantities may have led to difficulty in using the correct varying quantities and fixed quantities when constructing a linear equation for the situation (item 18).

\section{Discussion}

One purpose of this study was to explore the relationship between conceptual knowledge and procedural knowledge in the teaching of linear equations in two variables to community college students. The study compared the performance of a group of community college students instructed with a quantitative reasoning emphasis (ending with one day of procedural instruction) to the performance of a group of community college students instructed entirely with a procedural skill emphasis on the same unit assessment. The results of the analysis showed that there was no statistical difference in the performance of the groups on the procedural problems but that the intervention group had a greater performance on the conceptual problems. This supports two claims.

First, a quantitative reasoning emphasis with community college students followed by a short procedural skill instruction can result in greater conceptual understanding while also maintaining procedural skill proficiency compared to a procedural skill only instructional emphasis. This result is consistent with other research on quantitative reasoning in other learning situations (Thompson \& Thompson, 1992; Carlson et al., 2013; Ellis, 2007a; Simon \& Blume, 1994; Lobato \& Thanheiser, 2002) and recommendations that suggest procedural fluency can be supported through instruction focused on conceptual understanding (NCTM, 2014). Therefore, this exploratory study demonstrates that it is possible to address both conceptual understanding and procedural skill with community college students by focusing on conceptual understanding and spending minimal time on instructing for procedural skill.

Second, a procedural skill only emphasis with community college students does not result in greater conceptual understanding (in fact less conceptual understanding) compared to a conceptual understanding instructional emphasis with minimal procedural instruction. If the traditional procedural approach had conceptual instruction mixed with it or at the end of the procedural instruction the result may have been different.

Another purpose of the study was to examine the effects of a quantitative reasoning approach on community college students' ability to reason abstractly and quantitatively about linear relationships.

The first result of the analysis showed that the intervention group had a larger number of student responses that showed covariation reasoning ability than the comparison group on two of the items ( $44 \%$ vs. $23 \%$ on item 15 , and $31 \%$ vs. $16 \%$ on item 17). This is similar to Ellis' results (2007b) which showed that students engaged in quantitative reasoning made a greater number of correct global statements about linearity and a greater number statements of continuing phenomenon involving the co-varying nature of the quantities. The second result of the analysis showed that the intervention group had about the same number of student responses that showed correspondence reasoning ability compared to the comparison group on two of the items ( 2 vs. 1 on item 15, and 2 vs. 0 on item 17). These first two 
This is an author-produced, peer-reviewed version of this article. The final, definitive version of this document can be found online at School Science and Mathematics, published by Wiley. Copyright restrictions may apply. doi: 10.1111/ssm.12223

results demonstrate that the quantitative reasoning approach supported students' covariation reasoning ability but seemed to have had limited influence on correspondence reasoning (Jacobsen, 2014). Clearly, more work is needed to fully support both groups in their ability to express their reasoning, but this seems especially the case with correspondence reasoning.

The third result of the analysis revealed two misconceptions community college students may develop about linear relationships: the idea that a direct relationship between two quantities implies linearity and the idea that a constant rate (like one gallon every three seconds) is a varying quantity. These challenges associated with teaching and learning quantitative reasoning have been noted in other studies (Thompson, 1988; Lobato \& Thanheiser, 2002; Lobato et al., 2010; Dougherty \& Zbiek, 2010) and are important considerations for instructors aiming to support the development of students' quantitative reasoning. We discuss each in turn.

When students overgeneralize about mathematical relationships such as thinking that direct relationships are synonymous with linearity, we are forced to step back and reflect on the types of examples and problems students encountered during the unit. The examples and problems the intervention group encountered consisted of many examples and problems that were linear, in multiple representations, but only had one problem on a worksheet that was nonlinear in one representation - a table of values. This lack of exposure to nonlinear situations in multiple representations for the intervention group may have led to an overgeneralization about linearity. Incorporating the use of nonexamples and varied mathematical representations into a quantitative reasoning approach could support students in more deeply understanding linearity (NCTM, 2014).

Recall, 10 of the 31 responses in the comparison group and 22 of the 36 in the intervention group included claims that a constant rate is a varying quantity. Thus, over $60 \%$ of the students in the intervention group claimed that a constant rate was a varying quantity, whereas less than $1 / 3$ of the comparison group did so. This points to a potential developmental issue as students encounter fundamental ideas associated with rate that require students to join two varying quantities into a single quantity that does not vary, what is known as a composed unit in the proportional reasoning literature (Lamon, 2012). It has made us wonder if making explicit the meaning of a constant rate in terms of the quantities that are varying caused confusion for many of the students in the intervention group. Also because of the short duration, we suspect the intervention students did not have enough time to develop this particular idea. It seems the comparison group had an advantage here, but perhaps only in the sense that they had not been challenged to unpack their knowledge of rates in advance of the assessment. The development of proportional reasoning is foundational to the study of rate and is known to take considerable time. Weber, Ellis, Kulow, and Ozgur (2014) noted the importance of asking students to "identify particular quantities and how they intend to or imagine measuring those quantities" (p. 26). Incorporating this particular recommendation into a quantitative reasoning approach could support students in identifying the nature of quantities involved.

We can conclude from this study that small changes in instruction focused on conceptual understanding (in this case, conceptual understanding associated with linear relationships) can lead to significant differences in students' ability to demonstrate conceptual understanding compared to a predominately procedural approach. The results indicate that a quantitative reasoning approach shows promise in helping community college students develop covariation reasoning ability.

\section{Limitations}

The instructor who used the quantitative reasoning approach in this study had no prior experience with that instructional approach. A more experienced instructor may have been able to better manage the resistance by the students to the approach and better instruct the students using the approach - resulting in better performance on the unit assessment for the intervention group. It must also be noted that the quantitative reasoning approach was only used for one unit of the course (three 75-minute class periods) and started and ended in the middle of the course. Had the instructional approach been used for the entire duration of the course, the performance by the intervention group on the unit assessment may have been greater. Nevertheless, even with these limitations, more evidence of quantitative reasoning in the student responses of the intervention group was observed. 
This is an author-produced, peer-reviewed version of this article. The final, definitive version of this document can be found online at School Science and Mathematics, published by Wiley. Copyright restrictions may apply. doi: 10.1111/ssm.12223

\section{Implications for Future Study}

First, this study demonstrates the need for more teaching experiments that explore teaching mathematics for both conceptual understanding and procedural fluency. The study showed that more time can be spent on instructing for conceptual understanding without sacrificing procedural fluency. But how should the conceptual instruction be conducted, integrated, and assessed in conjunction with procedural instruction and assessment? Second, this study demonstrates the promise of a quantitative reasoning approach and a need to further develop and experiment with this instructional approach. Future experiments using the instructional approach need to rely on nonexamples of mathematical concepts and multiple representations of mathematical concepts to achieve deep understanding. The approach also needs to be improved to help students identify fixed and varying quantities in real world situations and especially help students recognize that a rate is a fixed quantity. How can instruction help students understand the difference between fixed and varying quantities in a real world situation and especially understand why a rate (as a composed unit) is a fixed quantity even though it is made by joining two varying quantities? Of course, this must be done with an eye to future mathematical work where rates of change are thought of as varying quantities in the study of calculus.

\section{References}

Belue, P.T. (2015). The impact of a quantitative reasoning instructional approach to linear equations in two variables on student achievement and student thinking about linearity. Master of Science Thesis, Boise, ID: Boise State University.

Blair, R. (2006). Beyond Crossroads: Implementing Mathematics Standards in the First Two Years of College. Memphis, TN: AMATYC.

Carlson, M.P., Jacobs, S., Coe, e., Larsen, S., \& Hsu, E. (2002). Applying Covariational Reasoning While Modeling Dynamic Events: A Framework and a Study. Journal for Research in Mathematics Education, 33(5), November, 352-378.

Carlson, M. P., Oehrtman, M., \& Moore, K. (2013). Precalculus Pathways to Calculus Student Workbook. Gilbert, AZ: Rational Reasoning, LLC.

Dougherty, B. J., \& Zbiek, R.M. (2010). Developing Essential Understanding of Number and Numeration for Teaching Mathematics in Prekindergarten-Grade 2. Reston, VA: National Council of Teachers of Mathematics.

Ellis, A.B. (2007a). The influence of reasoning with emergent quantities on students' generalizations. Cognition and Instruction, 25(4), 439-478.

Ellis, A.B. (2007b). A taxonomy for categorizing generalizations: Generalizing actions and reflection generalizations. Journal of the Learning Sciences, 16(2), 221-262.

Ellis, A.B. (2009). Patterns, Quantities, \& Linear Functions. Mathematics Teaching in the Middle School, 14(8) April, 482-491.

Fuson, K.C. (1988). Children's counting and concept of number. New York: Springer-Verlag Harel, G. (2001). The development of mathematical induction as a proof scheme: A model for DNR-based instruction. Manuscript submitted for publication.

Jacobson, E. (2014). Using covariation reasoning to support mathematical modeling. The Mathematics Teacher, 107(7), 515-519.

Karmiloff-Smith, A. (1992). Beyond modularity: A developmental perspective on cognitive science. Cambridge, MA; MIT Press.

Lamon, S. (2012). Teaching Fractions and Ratios for Understanding. Third Edition. Mahwah: Erlbaum.

Lappan, G., Fey, J.T., Fitzgerald, W.M., Friel, S.N., \& Phillips, E.D. (2009). Connected Mathematics 2, Moving Straight Ahead. Boston, MA: Pearson/Prentice Hall.

Leinward, S., Brahier, D.J., \& Huinker, D. (2014). Principles to Actions, Ensuring Mathematical Success for All. Reston, VA: NCTM.

Lobato, J., \& Thanheiser, E. (2002). Developing understanding of ratio-as-measure as a foundation for slope. In B. Litwiller \& G. Bright (Eds.), Making sense of fractions, ratios, and proportions: 2002 yearbook (pp. 162175). Reston, VA: National Council of Teachers of Mathematics.

Lobato, J., Ellis, A.B., \& Charles, R.I. (2010). Developing Essential Understanding of Ratios, Proportions \& Proportional Reasoning Grades 6-8. Reston, VA: National Council of Teachers of Mathematics.

Moore, K.C., \& Carlson, M.P. (2012). Students' images of problem contexts when solving applied problems. Journal of Mathematical Behavior, 31(1), 48-59. 
This is an author-produced, peer-reviewed version of this article. The final, definitive version of this document can be found online at School

Science and Mathematics, published by Wiley. Copyright restrictions may apply. doi: 10.1111/ssm.12223

Rittle-Johnson, B., \& Siegler, R.S. (2001). Developing Conceptual Understanding and Procedural Skill in Mathematics: An Iterative Process. Journal of Educational Psychology, 93(2), 346-362

Rittle-Johnson, B., Schneider, M., \& Star, J. R., (2015). Not a One-Way Street: Bidirectional Relations Between Procedural and Conceptual Knowledge of Mathematics. Educational Psychology Review, 27(4), 587-597.

Schwartz, D. L., \& Martin, T. (2004). Inventing to prepare for future learning: The hidden efficiency of encouraging original student production in statistics instruction. Cognition and Instruction, 22(2), 129-184.

Simon, M.A., \& Blume, G.W. (1994). Mathematical modeling as a component of understanding ratio-as-measure: A study of prospective elementary teachers. Journal of Mathematical Behavior, 13, 183-197.

Siegler, R. S., \& Stern, E. (1998). Conscious and unconscious strategy discoveries: A microgenetic analysis. Journal of Experimental Psychology: General, 127, 377-397.

Smith, T., Seshaiyer, P., Peixtoto, N., Suh, J.M., Bagshaw, G., Collins, Laurena K. (2013). Exploring Slope with Stairs and Steps. Mathematics Teaching in the Middle School, 18(6), February 2013, 370-377.

Thompson, P.W. (1988). Quantitative concepts as a foundation for algebraic reasoning: sufficiency, necessity, and cognitive obstacles. In M. Behr (Ed.), Proceedings of the annual meeting of the North American chapter of the international group for the psychology of mathematics education, vol. 1, 163-170.

Thompson, P. W., \& Thompson, A. G. (1992, April). Images of rate. Paper presented at the Annual Meeting of the American Educational Research Association, San Francisco, CA.

Thompson, P.W. (1994). The development of the concept of speed and its relationship to concepts of rate. In G. Harel \& J. Confrey (Eds.), The development of multiplicative reasoning in the learning of mathematics (pp. 179-234). Albany, NY: SUNY Press.

Trigsted, K., Bodden, K., \& Gallaher, R. (2014). Developmental Mathematics (pp. 12-1 to 12- 50). Boston, MA: Pearson Education.

Weber, E., Ellis, A., Kulow, T., \& Ozgur, Z. (2014). Six principles for quantitative reasoning and modeling. The Mathematics Teacher, 108(1), 24-29.

Table 1

Procedural Item Scaled Score Means and Standard Deviations

\begin{tabular}{|lcc|}
\hline \multicolumn{1}{|c}{$\begin{array}{c}\text { Unit Assessment } \\
\text { Item Number: Description }\end{array}$} & \multicolumn{2}{c|}{ Mean (Standard Deviation) } \\
\cline { 2 - 3 } & $\begin{array}{c}\text { Comparison } \\
(\mathrm{n}=34)\end{array}$ & $\begin{array}{c}\text { Intervention } \\
(\mathrm{n}=37)\end{array}$ \\
\hline 1: Plot an ordered pair on the coordinate plane & $0.95(0.12)$ & $0.86(0.26)$ \\
2: Find unknown coordinates of equation & $0.90(0.20)$ & $0.93(0.16)$ \\
3: Determine if ordered pair is solution & $0.98(0.09)$ & $0.99(0.04)$ \\
4: Find the slope of a line through two points & $0.92(0.19)$ & $0.79(0.32)$ \\
5: Graph a linear equation using intercepts & $0.73(0.32)$ & $0.71(0.30)$ \\
6: Find slopes of line parallel and perpendicular & $0.91(0.25)$ & $0.78(0.34)$ \\
7: Determine slope and y-intercept of equation & $0.79(0.32)$ & $0.71(0.33)$ \\
8: Graph a horizontal line & $0.86(0.29)$ & $0.77(0.35)$ \\
9: Determine if parallel, perpendicular, or neither & $0.62(0.44)$ & $0.56(0.42)$ \\
10: Graph line given point and slope & $0.80(0.34)$ & $0.65(0.38)$ \\
\hline
\end{tabular}


Table 2

Conceptual Item Scaled Score Means and Standard Deviations

\begin{tabular}{|lcc|}
\hline \multicolumn{1}{c}{ Unit Assessment } & \multicolumn{2}{c|}{ Mean (Standard Deviation) } \\
\cline { 2 - 3 } Item Number: Description & $\begin{array}{c}\text { Comparison } \\
(\mathrm{n}=34)\end{array}$ & $\begin{array}{c}\text { Intervention } \\
(\mathrm{n}=37)\end{array}$ \\
11: Identify fixed quantities in a situation & $0.54(0.30)$ & $0.85(0.27)$ \\
12: Identify variable quantities in a situation & $0.43(0.27)$ & $0.54(0.26)$ \\
13: Create table of values for a situation & $0.50(0.39)$ & $0.70(0.31)$ \\
14: Determine if a situation is linear & $0.94(0.20)$ & $0.89(0.32)$ \\
15: How does table tell you situation is linear? & $0.43(0.35)$ & $0.58(0.36)$ \\
16: Create a graph that models situation & $0.58(0.33)$ & $0.73(0.27)$ \\
17: How does graph tell you situation is linear? & $0.36(0.36)$ & $0.59(0.30)$ \\
18: Construct linear equation for situation & $0.48(0.32)$ & $0.64(0.17)$ \\
19: Explain numbers and variable in equation & $0.40(0.27)$ & $0.59(0.22)$ \\
20: Identify and explain quantity in situation & $0.21(0.23)$ & $0.64(0.36)$ \\
\hline
\end{tabular}

Table 3

Distribution of Scaled Score Means of Conceptual Items 11, 17, and 20

\begin{tabular}{|ccccccc|}
\hline $\begin{array}{c}\text { Scaled } \\
\text { Score } \\
\text { Measure }\end{array}$ & $\begin{array}{c}\text { Comparison } \\
(\mathrm{n}=34)\end{array}$ & $\begin{array}{c}\text { Intervention } \\
(\mathrm{n}=37)\end{array}$ & $\begin{array}{c}\text { Comparison } \\
(\mathrm{n}=34)\end{array}$ & $\begin{array}{c}\text { Intervention } \\
(\mathrm{n}=37)\end{array}$ & $\begin{array}{c}\text { Comparison } \\
(\mathrm{n}=34)\end{array}$ & $\begin{array}{c}\text { Intervention } \\
(\mathrm{n}=37)\end{array}$ \\
\hline Minimum & 0 & 0 & 0 & 0 & 0 & 0 \\
Quartile 1 & 0.33 & 0.66 & 0.25 & 0.25 & 0 & 0.25 \\
Median & 0.33 & 1 & 0.25 & 0.5 & 0.25 & 0.75 \\
Quartile 3 & 0.67 & 1 & 0.5 & 1 & 0.25 & 1 \\
Maximum & 1 & 1 & 1 & 1 & 0.75 & 1 \\
\hline
\end{tabular}

Table 4

Qualitative Analysis Category Student Response Counts

\begin{tabular}{|lcccc|}
\hline Qualitative Analysis Category & \multicolumn{2}{c}{ Item 15 } & \multicolumn{2}{c|}{ Item 17 } \\
\cline { 2 - 5 } & Comparison & Intervention & Comparison & Intervention \\
\hline Covariation Reasoning & 7 & 16 & 5 & 11 \\
Correspondence Reasoning & 2 & 1 & 2 & 0 \\
Beginning Quantitative Reasoning & 15 & 12 & 18 & 22 \\
Non-quantitative reasoning & 7 & 7 & 6 & 3 \\
Totals & 31 & $36^{*}$ & 31 & 36 \\
\hline
\end{tabular}

*There were 35 student responses, with one response coded in two categories 


\section{Figure 1}

\section{Procedural Item Scaled Score Means \& Overall Mean Bar Chart}

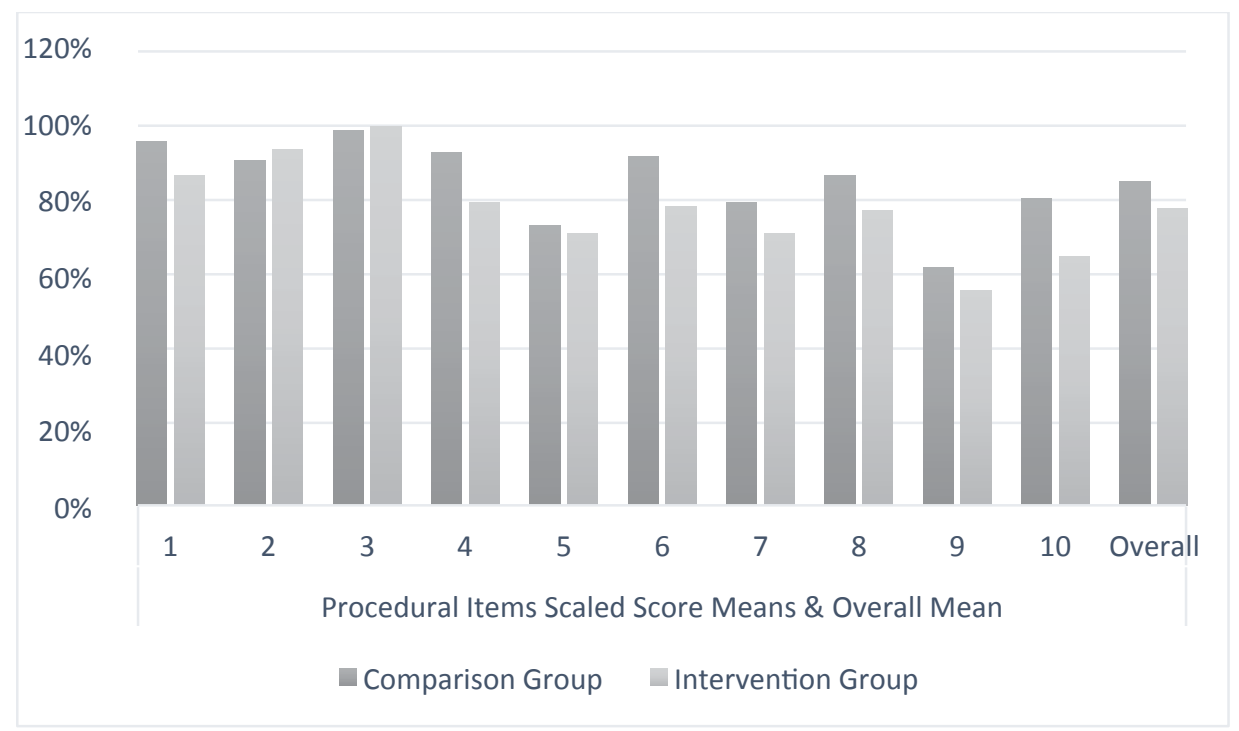

Figure 2

\section{Conceptual Item Scaled Score Means \& Overall Mean Bar Chart}

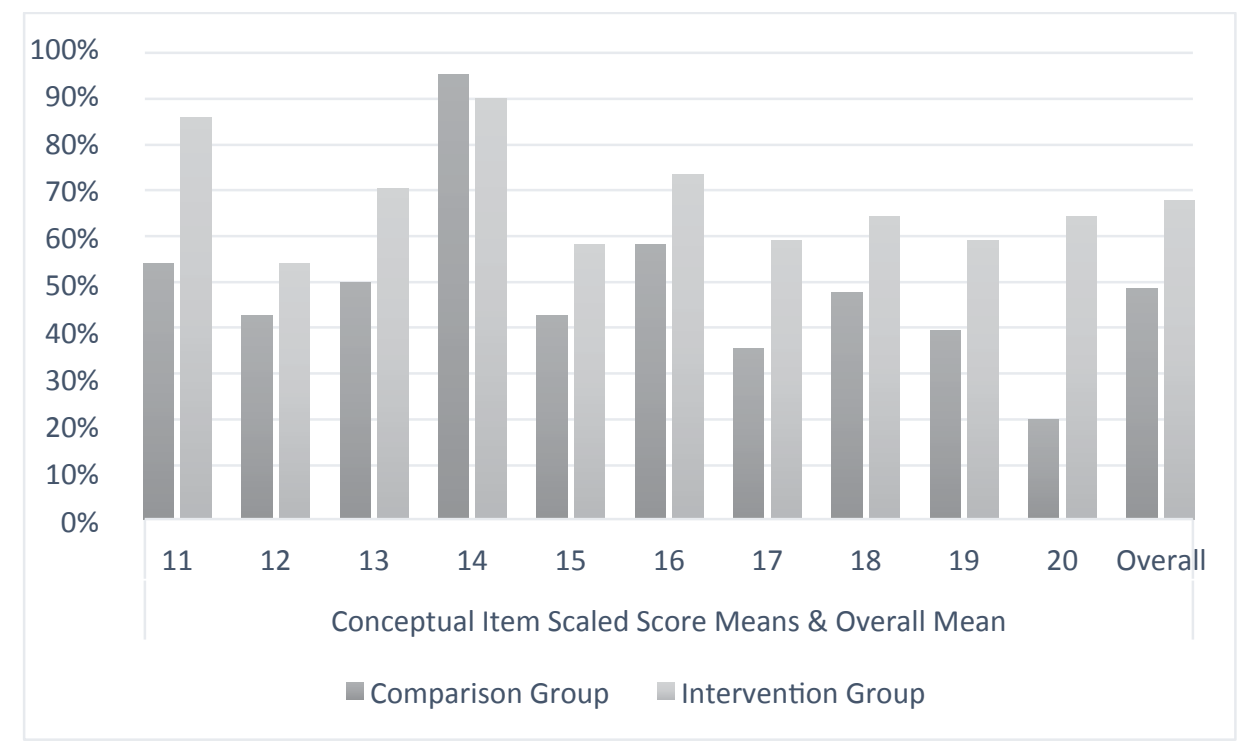

